\title{
Controle de Regimes de Dosagens e Ajuste de Parâmetros Farmacocinéticos
}

\author{
Vitor F. Ebertz ${ }^{1}$, Juliano V. Carvalho ${ }^{1}$, Rodrigo Goulart ${ }^{1}$, Cíntia O. Monticelli ${ }^{1,}$ \\ Rafael Linden ${ }^{1}$ \\ ${ }^{1}$ Instituto de Ciências Exatas e Tecnológicas - Universidade Feevale \\ Novo Hamburgo - RS - Brazil \\ \{vitorebertz,julianovc, rodrigo, cintiam, rafael.linden\} @ feevale.br
}

\begin{abstract}
This paper proposes a software of prediction and record of therapeutic drug dosing regimens, simulating concentration curves and adjusting patient pharmacokinetic parameters. The developed solution uses patient data provided by experts in the field. The proposed software, manages patient data, simulates the pharmacokinetic behavior of a drug in a treatment and applies the Bayesian method for individualization of drugs dosages. After testing the developed solution and comparing it with other software already adopted by academics and pharmacology professionals, it has found promising results.
\end{abstract}

Resumo. Este trabalho propõe um software para a previsão e registro de regimes de dosagens de substâncias terapêuticas, simulação de curvas de concentração e ajuste dos parâmetros farmacocinéticos de pacientes. A proposta desenvolvida utiliza dados de pacientes fornecidos por especialistas na área. O software desenvolvido, gerencia dados de pacientes, simula o comportamento farmacocinético de um fármaco em um tratamento e aplica o método Bayesiano para individualização das dosagens de fármacos. Após testar a solução desenvolvida e compará-la com outros softwares já adotados por acadêmicos e profissionais da farmacologia, verificou-se que os resultados obtidos são promissores.

\section{Introdução}

De acordo com Proost e Meijer (1992), a concentração de um fármaco no organismo deve ser mantida dentro de uma janela terapêutica para que a aplicação deste fármaco tenha o efeito planejado. Conforme Mehvar (1998), a janela terapêutica é a gama de valores de concentração entre o nível mínimo necessário para que o medicamento cause efeito $\left(\mathrm{C}_{\min }\right)$ e o nível máximo possível antes de apresentar toxicidade $\left(\mathrm{C}_{\max }\right)$.

Conforme Fuchs et al. (2013), o cálculo de regimes de dosagem individualizados requer um conjunto de habilidades e estudos que demanda tempo para os profissionais da área. Torna-se útil, portanto, o uso de programas de computador para o auxílio à decisão sobre dosagens de fármacos. Os mesmos autores também avaliam doze soluções disponíveis para uso clínico, todas em idioma estrangeiro, que incluem o software MwPharm, apontado como um dos mais apropriados para diversos casos. Apesar disso, é necessário treinamento para uso da interface do mesmo. O método bayesiano já está implementado em boa parte das soluções estudadas. Proost e Meijer 
(1992) definem o ajuste bayesiano como um método para aproximar parâmetros farmacocinéticos estimados para um dado indivíduo, considerando os dados populacionais e as concentrações medidas a partir de amostras de sangue do paciente. Este procedimento pode ser realizado a partir de qualquer quantia de coletas de concentração do fármaco, e as próximas coletas são usadas para realizar ajustes ulteriores.

Sheiner e Beal (1982) afirmam que a individualização do regime de dosagem é alcançada por meio de uma estimativa média populacional dos parâmetros farmacocinéticos, além de informações sobre a concentração da substância no paciente em questão. Partindo de uma estimativa inicial, são utilizados dados do paciente para adequar o modelo para cada indivíduo. O modelo inicial pode ser aprimorado pelo método bayesiano para uma aproximação mais precisa de cada caso, e tal método pode ser implementado em software.

Softwares de individualização do regime de dosagem já existem no mercado, porém as soluções pesquisadas e testadas para o desenvolvimento deste trabalho são todas estrangeiras, de uso complexo para usuários iniciantes. Além disso, estão divididas da seguinte maneira: (i) soluções comerciais, com capacidade de manter um banco de dados de pacientes, exibir simulações fidedignas e realizar os ajustes utilizando algoritmos variados; e (ii) soluções gratuitas, extremamente simplificadas, sem exibição de gráficos ou banco de dados de pacientes.

Por meio de pesquisas realizadas e bibliografia especializada, não foram encontrados softwares em português com a função de simular regimes de dosagens de fármacos e realizar os ajustes de parâmetros, apesar de tais programas existirem em idiomas estrangeiros. Tal lacuna foi o critério de motivação da presente pesquisa, qual seja a criação de uma solução disponível, em idioma português, na área da farmacocinética, para o cálculo individualizado de dosagens de fármacos.

Nesses termos, o foco da pesquisa é o desenvolvimento de um software com a função de auxiliar agentes de saúde na administração de fármacos, individualizando regimes de dosagem dos medicamentos a partir de dados dos pacientes. Os resultados do desenvolvimento foram validados por especialistas na área e comparados com outras soluções já disponíveis no mercado.

Este artigo está dividido em cinco seções. Além desta introdução, a segunda seção aborda a área de Farmacocinética e os conceitos essenciais para facilitar a compreensão do software proposto. A seção três explica o processo de desenvolvimento do software, bem como suas principais telas. A quarta seção aborda os testes realizados do software proposto (PKSoft) com os dados de três pacientes e o fármaco vancomicina. A última seção faz as considerações finais a respeito do trabalho que vem sendo desenvolvido.

\section{Estimativa de Parâmetros Farmacocinéticos}

Conforme Bauer (2014), clearance ou eliminação, expresso em unidades de volume por unidade de tempo, é uma medida da taxa com que o organismo elimina o fármaco do sangue. Pode ser utilizado para calcular a dose de manutenção necessária para a obtenção de uma determinada concentração em estado estacionário, sendo considerado, portanto, o parâmetro farmacocinético mais importante. 
O volume de distribuição é outro essencial parâmetro farmacocinético. Ele representa o volume de sangue necessário para conter todo o fármaco no corpo na concentração em que este se encontra. Bauer (2014) afirma que o volume de distribuição é igual ao volume de sangue contido no organismo do paciente, e é utilizado para calcular a dose de carga, ou loading dose. A dose de carga é a dose necessária para, após uma aplicação, alcançar dada concentração em estado estacionário. Como o volume de distribuição não é conhecido antecipadamente, é necessário estimar o parâmetro com base nos dados do paciente, de modo que as primeiras doses não gerarão um estado estacionário, podendo este ser atingido somente após a coleta de concentrações do plasma sanguíneo.

\subsection{Modelos farmacocinéticos}

Os modelos matemáticos utilizados para a sugestão de dosagens são baseados em parâmetros farmacocinéticos que, por sua vez, são calculados a partir dos parâmetros fisiológicos diretamente obtidos dos pacientes. Contudo, as funções utilizadas para chegar até os parâmetros farmacocinéticos são imprecisas em função da variabilidade existente entre os indivíduos. É por este motivo que devem ser empregados métodos de ajuste que levem em consideração a imprecisão dos cálculos iniciais (Sheiner et al., 1979). Um modelo farmacocinético simplificado com fins didáticos é fornecido por Mould e Upton (2012), ilustrado pela Equação 1:

$$
C(t)=\frac{\text { Dose }}{V} e^{\frac{-C l t}{V}}(1)
$$

No modelo apresentado, $\mathrm{C}(\mathrm{t})$ representa a concentração, variável dependente, em função do tempo, variável independente. Dose, $\mathrm{V}$ e $C l$ são as constantes que representam os parâmetros do modelo, a saber, respectivamente, dosagem, volume de distribuição e clearance.

\subsection{Ajuste de parâmetros}

Conforme Winter (2004), é possível realizar o ajuste de um dos dois parâmetros: clearance ou volume de distribuição, a partir de uma única coleta de plasma sanguíneo para obtenção da concentração de um fármaco. Existem diversas fórmulas para o ajuste dos parâmetros após a coleta de concentrações, e podem ser utilizadas de forma direta, inserindo os valores obtidos em uma função e observando os resultados. Como exemplo, o clearance de um fármaco para um determinado paciente pode ser ajustado após a obtenção de um valor real para a constante de eliminação, conforme a equação 2 , utilizando uma concentração medida no lugar de Cssı. O novo clearance pode então ser calculado através da equação $C l=K * V$, com $K$ representando a constante de eliminação e $V$ representando o volume de distribuição. Ainda assim, esse ajuste não é tão preciso quanto os métodos iterativos, como o ajuste bayesiano.

$$
C_{S S 1}=\frac{\frac{S * F * \text { Dose }}{V}}{1-e^{-K * \tau}} e^{-K * t_{1}}
$$

$\mathrm{Na}$ Equação 2, $C_{s S 1}$ representa a concentração em estado estacionário, $S * F$ representa a quantidade de fármaco que atinge a circulação, $V$ é o volume de distribuição, $K$ é a constante de eliminação e $\tau$ representa o intervalo entre doses e a variável $t_{1}$ representa o momento no tempo em que a concentração está sendo calculada (Winter, 2004). 
A abordagem indicada por Sheiner et al. (1979), sinaliza que uma estrutura estatística deve conter o modelo matemático do fármaco. Uma vez que, os resultados do modelo em uma estrutura estatística são considerados como o valor da moda de uma distribuição normal de probabilidade, a fase de ajuste requer a aplicação do cálculo bayesiano para aprimorar a distribuição de frequências dos parâmetros do modelo. Os valores dos parâmetros são então substituídos pelo valor da moda da nova distribuição.

$$
O B J_{\text {Bayes }}=\sum_{i=1}^{n} \frac{\bar{P}_{l}-\widehat{P}_{l}}{\sigma_{P_{i}}^{2}}+\sum_{i=1}^{m} \frac{\left(C P_{i}-\widehat{C P}_{l}\right)^{2}}{\sigma_{C P_{i}}^{2}}
$$

Na fórmula apresentada pela Equação 3, $\bar{P}$ representa os parâmetros iniciais estimados, enquanto $\hat{P}$ são todos os parâmetros do paciente. $\widehat{C P}$ é o conjunto de concentrações plasmáticas do fármaco obtidas diretamente do paciente, e $C P$ são as concentrações inicialmente previstas pelo modelo. A variância interindividual do parâmetro $i$ é representada por $\sigma_{P_{i}}^{2}$, e $\sigma_{C P_{i}}^{2}$ representa a variância do erro residual da concentração $i$. O valor da moda da nova distribuição consiste na minimização da fórmula acima. $\mathrm{O}$ ajuste Bayesiano permite a utilização de um número reduzido de amostras, sendo que apenas uma concentração mensurada já é suficiente para realizar um ajuste nos parâmetros cinéticos e na curva de concentração.

\section{Modelagem e Desenvolvimento}

O objetivo final da presente pesquisa é a elaboração de um software, doravante denominado PKSoft, capaz de executar as funções de cálculo de regime de dosagens, simulação de curvas de concentração e ajuste individualizado de parâmetros farmacocinéticos.

\subsection{Requisitos}

Ao longo do desenvolvimento desta pesquisa, durante reuniões com a equipe do Grupo de Pesquisa em Farmacocinética de uma universidade foram investigados softwares similares. Esses softwares foram selecionados com base no conhecimento prévio desse grupo na área de Farmacocinética. Posterior à análise deles, foram obtidos os requisitos funcionais pertinentes ao software proposto, os quais são exibidos na Tabela 1.

Tabela 1 - Requisitos funcionais levantados para desenvolvimento do PKSoft.

\begin{tabular}{|c|c|c|c|c|c|}
\hline $\begin{array}{l}\text { RF0 } \\
1\end{array}$ & Cadastrar novos pacientes. & $\begin{array}{l}\text { RF0 } \\
4 \\
\end{array}$ & Alterar regime de dosagens. & $\begin{array}{l}\text { RF0 } \\
7 \\
\end{array}$ & $\begin{array}{l}\text { Simular curvas de } \\
\text { concentração. }\end{array}$ \\
\hline $\begin{array}{l}\text { RF0 } \\
2\end{array}$ & $\begin{array}{l}\text { Alterar dados pessoais dos } \\
\text { pacientes. }\end{array}$ & $\begin{array}{l}\text { RF0 } \\
5\end{array}$ & Acessar histórico do paciente. & $\begin{array}{l}\text { RF0 } \\
8\end{array}$ & Cadastrar concentração medida. \\
\hline $\begin{array}{l}\text { RF0 } \\
3\end{array}$ & Criar regime de dosagens. & $\begin{array}{l}\text { RF0 } \\
6\end{array}$ & $\begin{array}{l}\text { Cadastrar dosagem de } \\
\text { fármaco. }\end{array}$ & $\begin{array}{l}\text { RF0 } \\
9\end{array}$ & $\begin{array}{l}\text { Ajustar parâmetros } \\
\text { farmacocinéticos do paciente. }\end{array}$ \\
\hline
\end{tabular}

\subsection{Interface Gráfica}

A interface gráfica foi desenvolvida a partir da biblioteca Shiny (pacote da linguagem R), utilizando-se a aparência padrão da biblioteca. As telas do programa são acessadas a partir de um menu superior, e no próprio fluxo do programa o usuário é direcionado para a tela seguinte através de botões. A tela inicial, Pacientes (Figura 1), contém as informações de todos os pacientes cadastrados e dos tratamentos (Figura 2) em andamento para estes. 


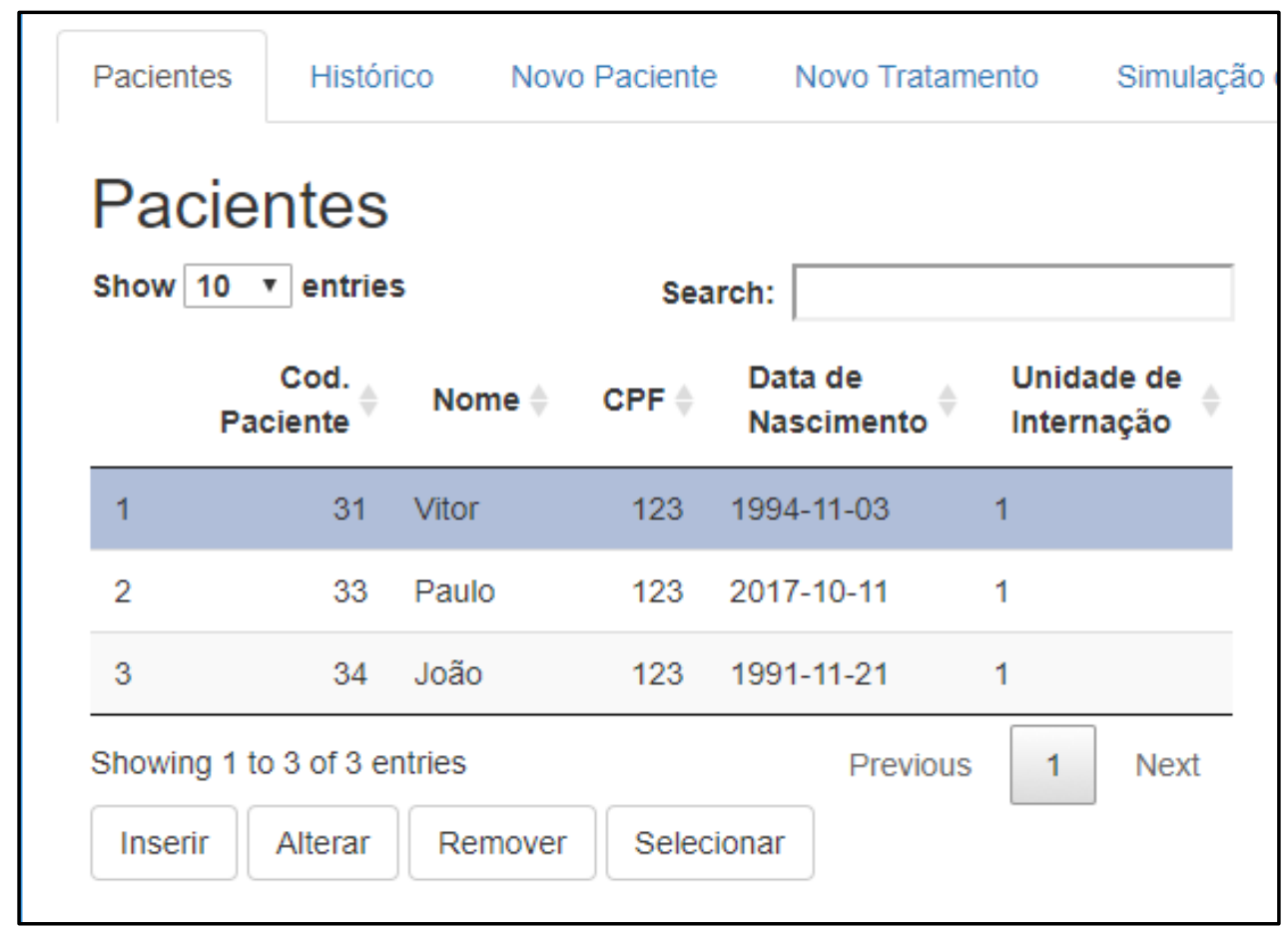

Figura 1 - Tela de registros de pacientes do PKSoft.

A partir da tela inicial (Figura 1), o usuário pode ser direcionado para as demais conforme a ação desejada.

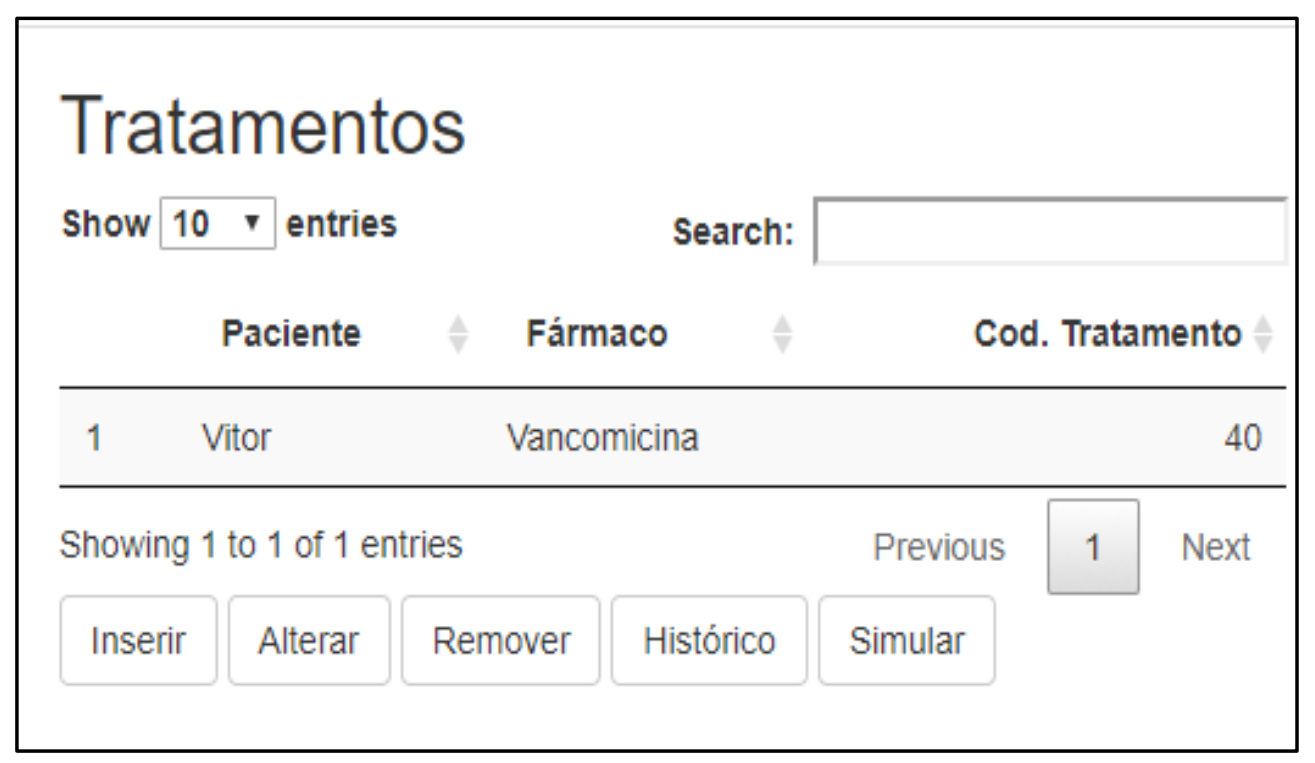

Figura 2 - Tela de registros de tratamentos de um paciente.

\subsection{Simulação de concentrações}

A tela de Simulação, representada na Figura 3, foi disposta da seguinte maneira: o gráfico contendo as curvas de simulação logo abaixo do título da página; os botões de ação abaixo; os campos exibindo a dose calculada e a concentração de vale prevista mais à esquerda, para referência; no centro, estão os campos editáveis contendo a dose utilizada, a concentração de vale, a duração da infusão do medicamento, o intervalo entre doses, a quantidade de doses administradas, bem como a data e hora de início do 
regime de dosagens; e à direita, os dados dos pacientes juntamente com os parâmetros farmacocinéticos, estes na última coluna divididos entre estimativas iniciais e parâmetros ajustados.

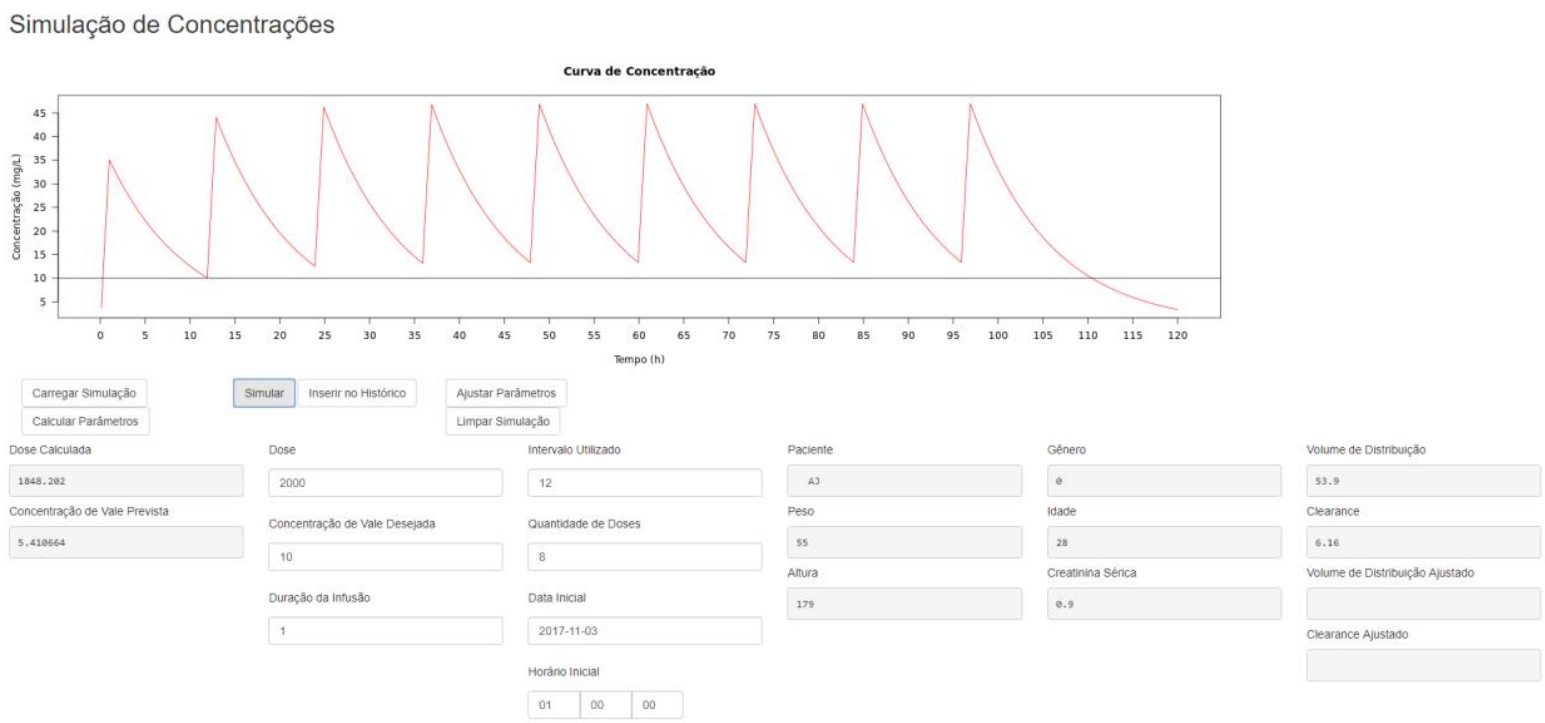

Figura 3 - Tela completa de simulação de concentrações do PKSoft.

A Figura 4 exibe o gráfico de simulação da concentração das doses, com as curvas de concentração atingindo o pico a cada nova dose, decaindo em seguida conforme a fórmula de concentração. A linha reta no gráfico representa a concentração de vale desejada, que é o valor mínimo esperado para as concentrações.

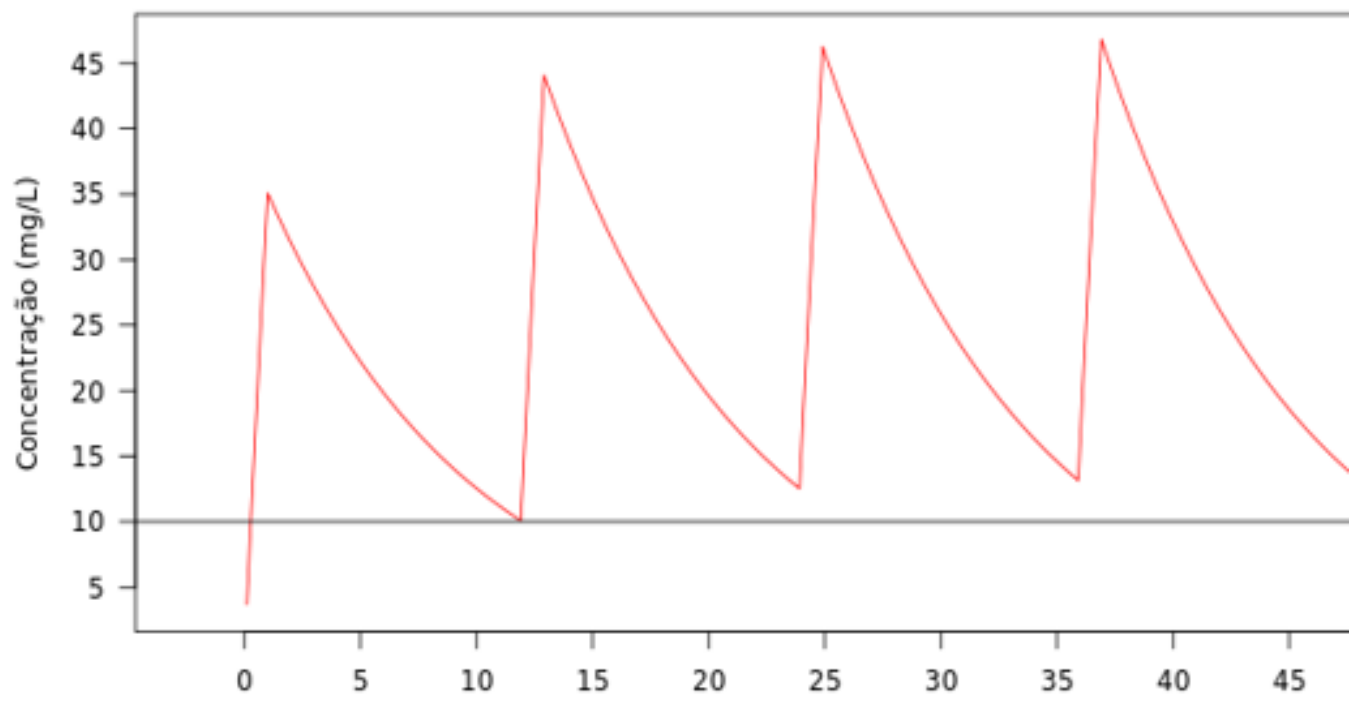

Figura 4 - Curvas de concentração das doses de um Fármaco. 
A Figura 5 exibe os campos referentes ao cálculo da dose. No lado esquerdo, o campo Dose Calculada exibe a dose exata que deve ser administrada para chegar à concentração ideal. Ao lado direito, está posicionado o campo Dose, que receberá a dose que está disponível e será utilizada pelo agente de saúde. Os campos Intervalo Utilizado, Quantidade de Doses e Data Inicial servem para gerar um regime de dosagem referente à dosagem proposta.

\begin{tabular}{l} 
Carregar Simulação \\
Calcular Parâmetros \\
Dose Calculada \\
Concentração de Vale Prevista \\
5.410664 \\
\hline
\end{tabular}
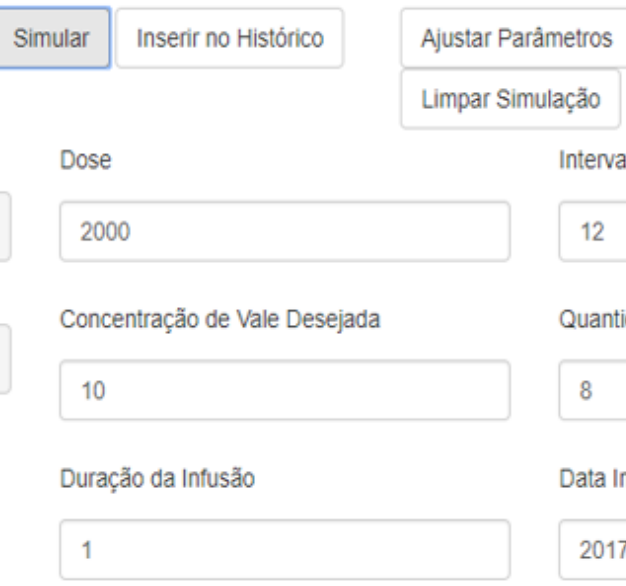

Figura 5 - Campos utilizados no cálculo de dosagem do PKSoft.

A Figura 6 exibe, além dos dados iniciais do paciente, os parâmetros farmacocinéticos calculados. Os quatro campos da direita (Volume de Distribuição, Clearance, Volume Ajustado e Clearance Ajustado) são referentes aos parâmetros, sendo os dois superiores referentes à estimativa inicial, e os dois inferiores ao cálculo bayesiano.
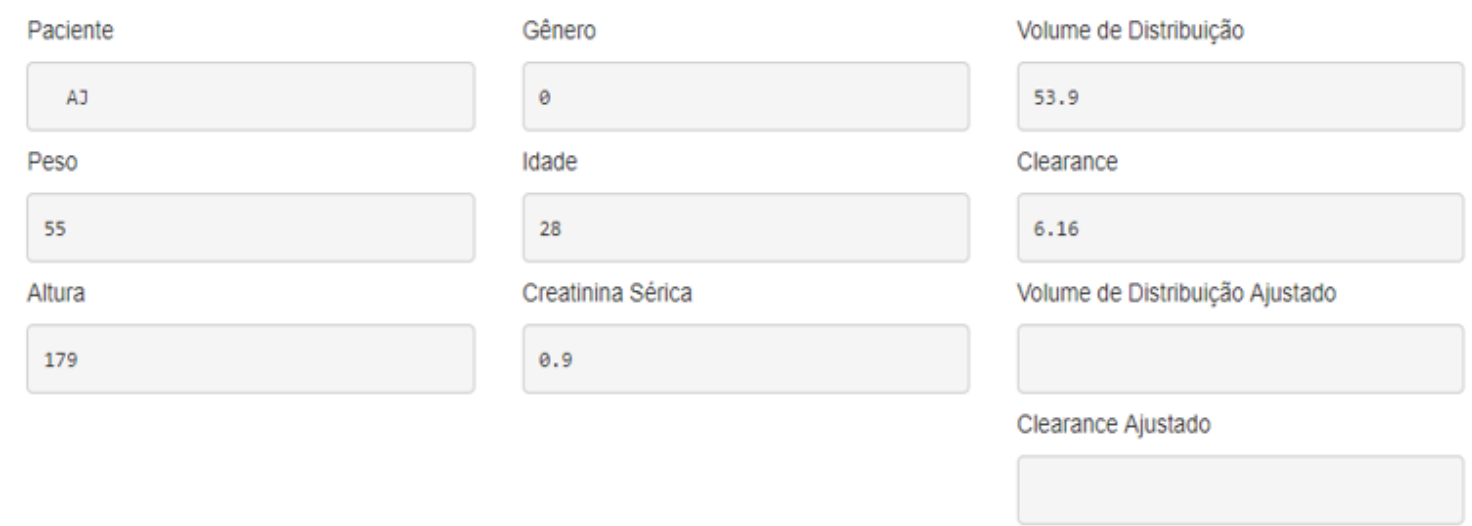

Figura 6 - Dados fisiológicos do paciente e seus parâmetros farmacocinéticos.

A concentração de vancomicina foi calculada utilizando a fórmula exibida na Equação 4:

$$
C_{t s S}=\frac{\text { Dose }}{C l} * \frac{1-e^{-k}}{\left(1-e^{-k t}\right)\left(e^{-k(t-1)}\right)}
$$


onde $C_{t s s}(\mathrm{mg} / \mathrm{L})$ é a concentração resultante, Dose $(\mathrm{mg})$ representa a dose inicial, $C l(\mathrm{mg} / \mathrm{L} / \mathrm{h})$ representa o Clearance de vancomicina, $k$ representa o parâmetro $K$, que é o resultado da divisão do Clearance pelo volume de distribuição, e $t$ representa o tempo em horas. O resultado da fórmula para cada ponto no tempo é armazenado em um vetor que representa a curva de concentração para uma dose.

\subsection{Ajuste Bayesiano}

Em virtude de seu enfoque em estatística e pesquisa científica, foi escolhida a linguagem de programação R para desenvolvimento do PKSoft. Esta linguagem dispõe de uma função para otimização de parâmetros, executada a partir do comando optim(). A entrada da função é composta da equação cujo resultado se pretende minimizar (Equação 3); as estimativas iniciais dos parâmetros a ajustar (parâmetros farmacocinéticos calculados a partir das fórmulas populacionais); o desvio padrão dos parâmetros; a massa de dados contendo os resultados do modelo e os resultados concretos. A função de ajuste Bayesiano utiliza os dados de uma simulação já cadastrada para o paciente especificado.

Os parâmetros farmacocinéticos ajustados através do método Bayesiano serão o Volume de Distribuição e o Clearance. Ambos os parâmetros são retornados como a saída da função optim(). Após o ajuste dos parâmetros, o PKSoft utiliza a função do cálculo das curvas de concentração, utilizando os novos parâmetros como entrada. Caso o usuário não tenha cadastrado nenhuma concentração para o paciente, ao utilizar a função de ajuste Bayesiano esta retornará a mesma curva da simulação inicial.

\section{Resultados e Validação}

A Tabela 2 exibe uma comparação direta entre o modelo dos especialistas do Grupo de Pesquisa em Farmacocinética, o software JPKD e o PKSoft. O Modelo dos especialistas está implementado em uma planilha excel e é amplamente utilizado em aulas do curso de Farmácia de uma universidade. O software de farmacocinética JPKD é gratuito e não permite a geração da curva de concentração, apenas o valor dos parâmetros após o ajuste bayesiano.

Tabela 2 - Comparativo dos parâmetros farmacocinéticos calculados pelo PKSoft com outras soluções.

\begin{tabular}{|c|c|c|c|c|c|c|c|c|c|c|c|c|}
\hline \multirow[b]{2}{*}{ Método } & \multicolumn{4}{|c|}{ Paciente AB } & \multicolumn{4}{|c|}{ Paciente CD } & \multicolumn{4}{|c|}{ Paciente EF } \\
\hline & $\mathrm{Cl}$ & $\begin{array}{c}\mathrm{Cl} \\
\text { Bayes }\end{array}$ & $\mathbf{v}$ & $\begin{array}{c}\mathrm{V} \\
\text { Bayes }\end{array}$ & $\mathrm{Cl}$ & $\begin{array}{c}\mathrm{Cl} \\
\text { Bayes }\end{array}$ & $\mathbf{v}$ & $\begin{array}{c}\mathbf{V} \\
\text { Bayes }\end{array}$ & $\mathrm{Cl}$ & $\begin{array}{c}\mathrm{Cl} \\
\text { Bayes }\end{array}$ & $\mathbf{v}$ & $\begin{array}{c}\mathrm{V} \\
\text { Bayes }\end{array}$ \\
\hline PKSoft & 4.70 & 5.23 & 53.90 & 47.47 & 5.60 & 3.73 & 83.30 & 89.50 & 1.84 & 1.65 & 73.5 & 74.80 \\
\hline $\begin{array}{l}\text { Modelo } \\
\text { Esp. }\end{array}$ & 4.63 & 5.23 & 53.90 & 47.47 & 5.59 & 3.73 & 83.30 & 88.97 & 1.84 & 1.66 & 73.5 & 74.67 \\
\hline JPKD & - & 5.21 & - & 47.74 & - & 3.72 & - & 87.72 & - & 1.65 & - & 72.68 \\
\hline
\end{tabular}

A partir da Tabela 2 é possível identificar que os parâmetros farmacocinéticos iniciais calculados pelo PKSoft são similares àqueles gerados pelos especialistas. Não é possível fazer a comparação com o JPKD, pois esse software apenas exibe os parâmetros ajustados. Após o ajuste bayesiano, os parâmetros calculados pelo PKSoft diferem em um nível considerado aceitável pelos especialistas em relação aos outros 
softwares. O software foi implementado somente para o modelo do fármaco Vancomicina. Modelos de outros fármacos estão em fase de implementação.

\section{Considerações finais}

O software PKSoft atingiu seus primeiros objetivos. Manteve o cadastro dos pacientes e seus registros de doses e concentrações. Demonstrou ser capaz de realizar os cálculos de dosagem para o fármaco vancomicina e ajustou individualmente as curvas de simulação de concentrações.

A contribuição deste trabalho está no desenvolvimento do software PKSoft, disseminando o conhecimento necessário para a produção de tecnologia relacionada à área da farmacologia, com base científica e auxílio de especialistas na área. Além disso, preenche a lacuna de softwares em português para a individualização do regime de dosagem e concentração de fármacos.

As possibilidades de desenvolvimento futuro são amplas, as principais são: a inclusão de outros modelos de fármacos no software e a possibilidade de editar e adicionar modelos novos. O PKSoft faz parte de um projeto de farmacoterapia personalizada, que pretende utilizar o software para realizar os cálculos de dosagem, com a ideia de ser integrado com outras soluções para então automatizar e registrar o momento exato da administração dos fármacos. Um dos próximos passos previstos será sua aprovação no Comitê de Ética em Pesquisa, a fim de garantir experimentos futuros com seres humanos.

\section{Referências}

BAUER, Larry A. Applied Clinical Pharmacokinetics. [Nova Iorque]: McGraw-Hill Education, 3 ed. 2014.

FUCHS, Aline; CSAJKA, Chantal; THOMA, Yann; BUCLIN, Thierry; WIDMER, Nicolas. Benchmarking Therapeutic Drug Monitoring Software: A Review of Available Computer Tools, Clinical Pharmacokinetics, v. 52, n. 1, p. 9-22, jan. 2013.

MEHVAR, Reza. Pharmacokinetic-based design and modification of dosage regimens. American Journal of Pharmaceutical Education, Des Moines, v. 63, n. 2, p. 189-195, 1998.

MOULD, Dr.; UPTON, Rn. Basic Concepts in Population Modeling, Simulation, and Model-Based Drug Development, CPT Pharmacometrics \& Systems Pharmacology, v.1, n. 9, ed. 6, 2012.

PROOST, Johannes H.; MEIJER, Dirk K. F. MW/Pharm, an integrated software package for drug dosage regimen calculation and therapeutic drug monitoring. Computers in Biology and Medicine, v. 22, n. 3, p. 155-163, may. 1992.

SHEINER, Lewis B.; BEAL, Stuart L. Bayesian individualization of pharmacokinetics: Simple implementation and comparison with non-Bayesian methods, Journal of Pharmaceutical Sciences, Nova Iorque, v. 17, n. 12, p. 1344-1348, dez. 1982.

SHEINER, Lewis B.; BEAL, Stuart; ROSENBERG, Barr; MARATHE, Vinay V. Forecasting individual pharmacokinetics. Clinical Pharmacology \& Therapeutics, v. 26, n. 3, p. 294-305, set. 1979. 
WINTER, Michael E. Basic Clinical Pharmacokinetics. Baltimore: Lippincott Williams \& Wilkins, 4 e. 2004. 